\section{Avaliação da tomografia computadorizada e da cisternotomografia computadorizada com Lopamidol no topodiagnóstico da fístula liquórica e comparação com os achados cirúrgicos}

\author{
Roberto E. S. Guimarães' ${ }^{1}$, Helena M.G. Becker², \\ Celso G. Becker ${ }^{3}$, Paulo Fernando Tormin Borges \\ Crosara', Gustavo C. Anjos ${ }^{5}$, Letícia P. Franco 6
}

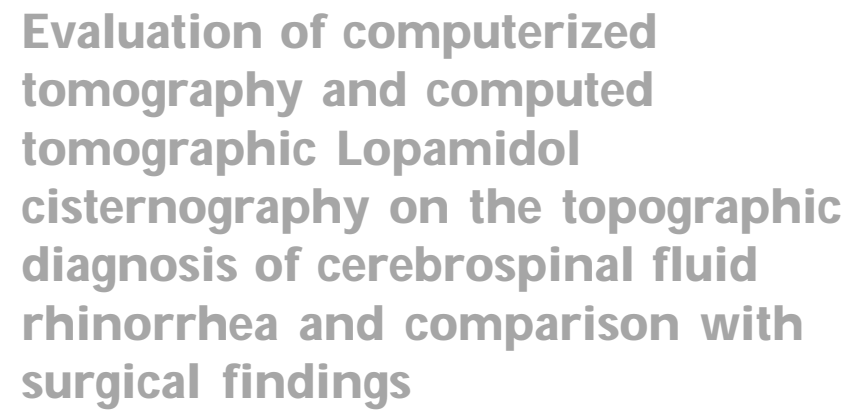

Resumo / Summary

D esde que a abordagem por via microendoscópica nasal se tornou uma rotina no tratamento da fístula liquórica da base anterior do crânio, a necessidade do topodiagnóstico preciso aumentou grandemente. A localização pré-operatória da fistula com métodos de imagem torna 0 ato cirúrgico mais rápido e direcionado. Objetivo: 0 objetivo deste estudo é avaliar a eficácia da cisternotomografia (CTG) e da tomografia computadorizada (TC) na localização da fístula liquórica de acordo com os achados cirúrgicos. Forma de Estudo: Observacional coorte com corte transversal. Material e Método: Quarenta e seis pacientes com diagnóstico clínico de fístula liquórica foram avaliados com TC, e destes, 38 também foram submetidos à CTG contrastada com Lopamidol. Resultado: A cisternotomografia apontou corretamente o local exato da fístula em $71 \%$ dos casos enquanto a tomografia o fez em $52 \%$ destes. A cisternotomografia foi importante na localização da fístula em $84,15 \%$. Conclusão: Ainda não há um método considerado padrão de referência no topodiagnóstico da fístula liquórica. Nesse propósito a CTG com Lopamidol é um método valoroso.
Palavras-chave: fístula liquórica, rinoliquorréia, cisternotomografia, Lopamidol, tomografia computadorizada. Key words: cerebrospinal fluid fistula, rhinorrhea, computed tomographic cisternography, Lopamidol, computed tomography.

ince the microendoscopic nasal approach to treat the cerebrospinal fluid (CSF) rhinorrhea turned a routine, the need of correct topographic diagnosis was greatly increased. Aim: The aim of this study is to evaluate the efficiency of the computed tomographic cisternography (CTC) and computerized tomography (CT) to locate the CSF fistula according with surgical finds. Study Design: Observacional cohort with transversal cut. Material and Method: Forty-six patients with clinical diagnosis of CSF rhinorrhea were accessed by CT. Results: Thirty-eight of them were also accessed by CTC with Lopamidol. CTC pointed the exact site of fluid escape in $71 \%$ of the cases and CT in $52 \%$. CTC helped to find the site of CSF rhinorrhea in $84,15 \%$ of the evaluated cases. Conclusion: There is not a gold standard method to access the site of CSF rhinorrhea yet. Lopamidol CTC is a worthy method in this purpose.

\footnotetext{
1 Professor Adjunto do Departamento de Otorrinolaringologia da Faculdade de Medicina da U.F.M.G

2 Professora Adjunta do Departamento de Otorrinolaringologia da Faculdade de Medicina da U.F.M.G

${ }_{3}$ Professor Assistente do Departamento de Otorrinolaringologia da Faculdade de Medicina da U.F.M.G ${ }_{4}^{4}$ Médico otorrinolaringologista, Doutorando-U.F.M.G.

${ }^{5}$ Médico residente do serviço de otorrinolaringologia do Hospital das Clínicas da UFMG

${ }^{6}$ Médica residente do serviço de otorrinolaringologia do Hospital das Clínicas da UFMG. Instituição: Departamento de O ftalmologia, O torrinolaringologia e Fonoaudiologia da UFMG. Endereço para Correspondência: Av. Pasteur, 88 Sta. Efigênia Belo Horizonte MG 30150-290. Tel $(0 \times x 31)$ 3222-2891

Artigo recebido em 28 de fevereiro de 2002. Artigo aceito em 05 de maio de 2002.
} 


\section{INTRODUÇÃO}

A rinoliquorréia (RLR) foi reconhecida como uma condição anormal pela primeira vez em 1782 por Willis. ${ }^{1}$ Quanto a sua classificação, pode ser dividida em dois grandes grupos: traumáticas e não-traumáticas. As traumáticas são subdivididas em acidental, iatrogênicas, agudas e tardias. As não-traumáticas se dividem em com ou sem hipertensão liquórica associada. ${ }^{2}$

A presença de uma fístula liquórica $(\mathrm{FL})$ implica em um risco aumentado de meningite, que varia de 9 a $25 \%$, mesmo nos casos em que a fístula tenha cessado espontaneamente. ${ }^{3,4}$ Devido a essas complicações serem extremamente graves e determinarem altos índices de morbimortalidade mesmo na atualidade, o tratamento da FL desperta preocupação e interesse dos otorrinolaringologistas e neurocirurgiões.

Com o desenvolvimento da cirurgia microendoscópica nasal, o tratamento da FL pelo otorrinolaringologista se tornou menos agressivo que a via extracraniana, sendo ao mesmo tempo eficaz. ${ }^{5-7} \mathrm{~A}$ abordagem endonasal aumentou a necessidade de métodos de imagem capazes de demonstrar com fidedignidade o local do escape liquórico, já que sua localização precisa torna essa cirurgia mais direcionada e conseqüentemente ainda mais eficaz, rápida e segura.

Uma série de méto dos de imagem já foi utilizada no diagnóstico da $\mathrm{RLR}$, desde até radiografias simples dos seios da face que apresentavam $25 \%$ de sensibilidade, cisternografias com radioisótopos com $50 \%$ de sensibilidade, politomografias com $53 \%$ de sensibilidade ${ }^{8}$, tomografias, cisternotomografias com metrizamida, com $87 \%$ de sensibilidade em pacientes com RLR ativa ${ }^{8}$ e ressonância nuclear magnética. ${ }^{9-12}$ Dos méto dos citados, apenas os últimos três têm colaborado no topodiagnóstico da FL.5,9,10

Assim, apresentamos uma experiência de 46 casos de FL em que comparamos os resultados da tomografia simples, da cisternotomografia entre si, com os achados cirúrgicos.

\section{MATERIAL E MÉTODO}

Durante o período de janeiro de 1993 a abril de 1999, 46 pacientes, 23 homens e 23 mulheres, com suspeita de rinoliquorréia foram submetidos a tomografia computadorizada dos seios da face em incidência axial e coronal sem contraste. Destes pacientes, 38 também foram submetidos à cisternotomografia com injeção intratecal após punção lombar no nível de L3-L4 ou no nível de cisterna magna, de 10 $\mathrm{cm}^{3}$ de meio de contraste hidrossolúvel não-iônico Lopamidol. Para que o contraste atingisse as cisternas intracranianas, após a aplicação, os pacientes foram colocados em posição de prece maometana por alguns minutos. A seguir realizou-se TC no plano coronal, fazendo-se uma varredura por toda a região crivosa e teto dos seios etmóides e esfenóides. Em um dos pacientes foi realizada tomografia de ossos temporais devido a suspeita de otoliquorréia. A idade mínima foi de 4 anos, a máxima de 66 anos e a mediana, de 39 anos. Destes pacientes, 14 apresentaram meningite previamente ao tratamento $(30,4 \%)$. 0 tempo de aparecimento dos sintomas até o tratamento variou de 10 dias a 17 anos.

Nos pacientes operados a partir de 1997, durante 0 procedimento cirúrgico, foi utilizado como corante intra-tecal a fluoresceína. Com exceção de 7 pacientes que usaram solução hiperdensa, todos os outros fizeram uso da solução hipodensa de fluoresceína que teve seu uso proposto inicialmente pelo autor e que auxilia a localização e a confirmação da eficácia do vedamento da fístula no transoperatório e permite que o paciente seja colocado em posição cirúrgica imediatamente após a injeção intratecal do corante, o que diminui o tempo cirúrgico. ${ }^{5}$

\section{RESULTADOS}

D os 46 pacientes submetidos à tomografia não contrastada, 24 exames sugeriram o local da FL concordantemente com os achados cirúrgicos (52,17\%); em 15 pacientes a TC foi inconclusiva $(32,6 \%)$ e em 5 pacientes a localização da FL sugerida pela TC foi discordante do achado cirúrgico (10,8\%). Em 2 pacientes em que a TC sugeriu 0 local de drenagem não se localizou FL durante a cirurgia $(4,3 \%)$.

Dos 38 pacientes que foram submetidos à cisternotomografia, 27 tiveram evidenciada a fístula de forma concordante com a cirurgia (71,05\%), 4 exames foram divergentes dos achados cirúrgicos (10,5\%), 6 exames falharam em identificar o extravasamento do contraste para a cavidade nasossinusal (15,7\%) e 1 exame evidenciou o contraste em etmóide anterior esquerdo, porém o local da fístula não foi encontrado durante a cirurgia (2,6\%). Nos casos onde houve divergência entre a localização da FL pelos exames, CTG e ou pela TC, e o achado cirúrgico da fístula, esta se encontrava justamente no seio adjacente ao demonstrado pelo método de imagem em todos os casos.

Nos 38 pacientes que se submeteram aos dois métodos de diagnóstico, houve 24 diagnósticos concordantes, 9 casos em que a TC foi inconclusiva e a CTC foi conclusiva, 4 casos em que ambos os métodos foram inconclusivos, 2 casos em que a TC foi conclusiva e a CTC foi inconclusiva e um caso onde a localização sugerida pelos exames foi discordante, sendo que 0 achado cirúrgico confirmou que a CTC demonstrou corretamente o local da FL. (Tabela 1)

\section{DISCUSSÃO}

Acreditamos que a abordagem propedêutica deva seguir uma seqüência. A princípio utilizamos exames para confirmar a RLR: a mensuração da glicose na secreção nasal é um método fácil e não-invasivo que utilizamos de rotina, 
Tabela 1. Comparativo dos achados radiológicos com achado cirúrgico nos 38 pacientes que foram investigados com os dois métodos propedêuticos.

\begin{tabular}{lcc}
\hline & CTC com Lopamidol & TC \\
\hline Concordância radiológica com achado transoperatório & $27(71,05 \%)$ & $19(50 \%)$ \\
Localização transoperatória da rinoliquorréia em seio & $04(10,52 \%)$ & $04(10,52 \%)$ \\
paranasal adjacente ao demonstrado radiológicamente & & \\
Exame inconclusivo & $06(15,78 \%)$ & $14(36,84 \%)$ \\
Pacientes com fístula não localizada no transoperatório & $01(2,6 \%)$ & $01(2,6 \%)$ \\
\hline
\end{tabular}

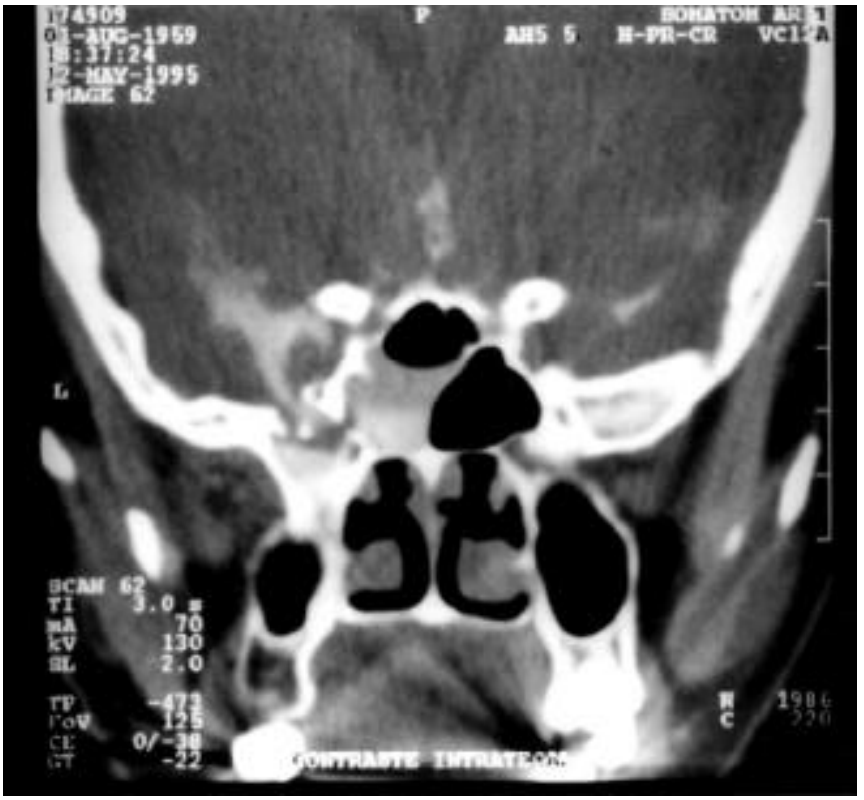

Figura 1. Cisternotomografia apresentando trauma nasal com fístula liquórica no etmóide posterior esquerdo (célula de Onodi) drenando para o seio esfenoidal.

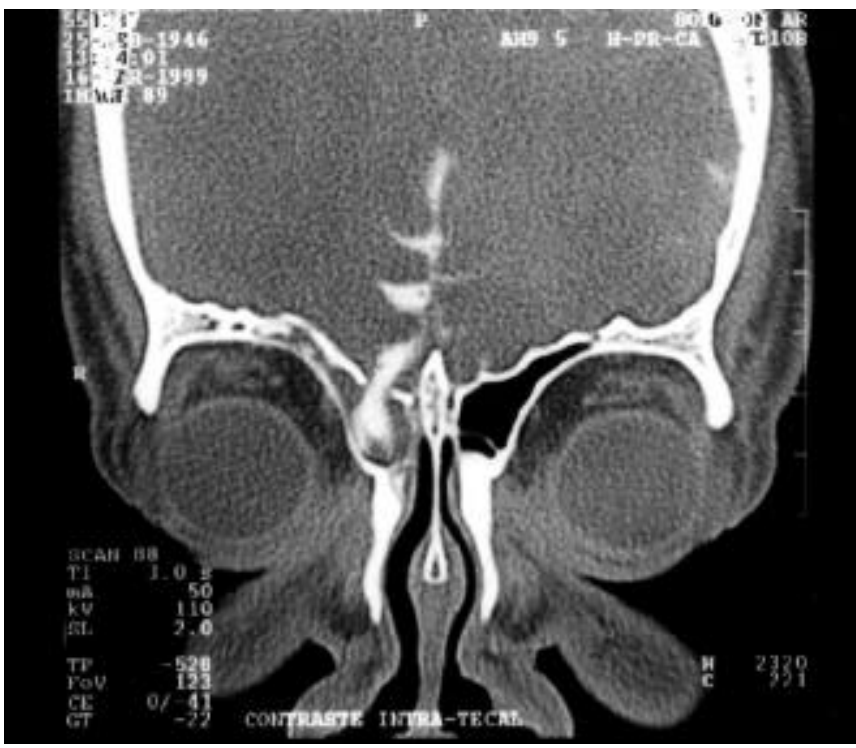

Figura 3. Cisternotomografia apresentando meningocele frontal direita.

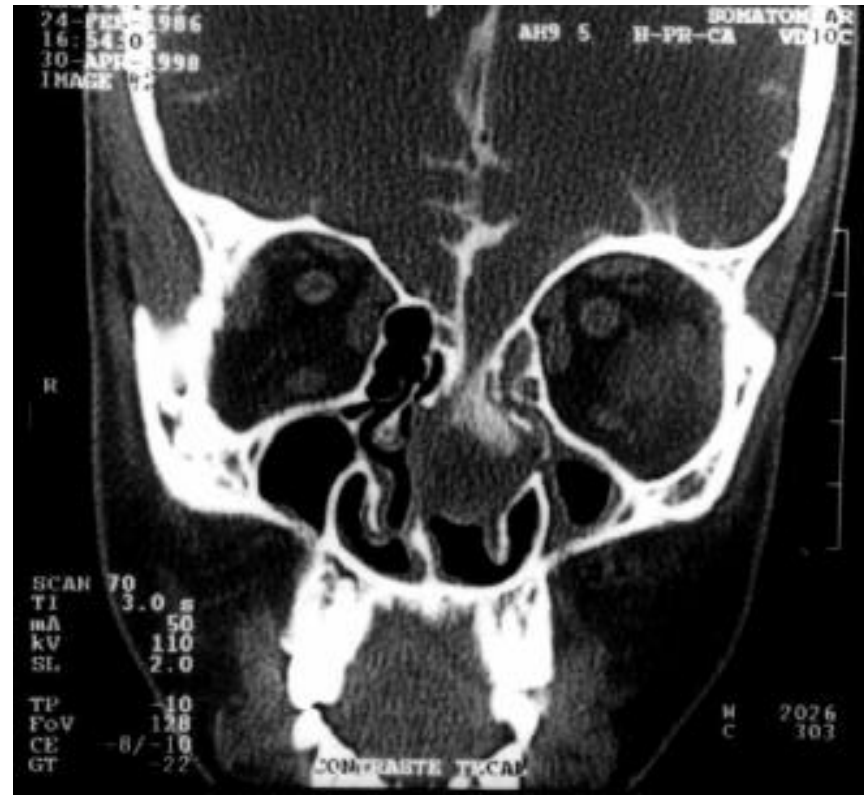

Figura 2. Cisternotomografia apresentando meningoencefalocele de lâmina crivosa esquerda.

sempre que possível, na qual valores acima de $30 \mathrm{mg} / \mathrm{dl}$ confirmam o diagnóstico de FL em pacientes com glicemia normal. ${ }^{13,14}$ Apesar de não estar disponível em nosso meio, é válido citar a pesquisa de b2 transferrina na rinorréia essa proteína é específica do líquor e da perilinfa, é o padrão de referência no diagnóstico $\mathrm{FL}$, e sua pesquisa é facilitada por ser necessária uma quantidade mínima de secreção para 0 diagnóstico. ${ }^{15,16}$

A técnica radiológica ideal para a localização acurada da fístula liquórica no pré-operatório é controversa. As maiores dificuldades diagnósticas encontram-se no subgrupo de pacientes que apresentam RLR intermitente ou inativa. ${ }^{10}$

0 papel da cisternotomografia com contraste hidrossolúvel não-iônico (metrizamida) na rinoliquorréia foi estabelecido primeiramente por Drayer. ${ }^{17}$ Subseqüentemente, este método se mostrou eficiente em várias publicações. ${ }^{18-20}$ Atualmente é considerado o método de escolha no topodiagnóstico da FL. Em nossa experiência, o método foi útil em identificar a RLR em $84,15 \%$ dos exames, sendo que em $71,05 \%$ 
dos casos o local preciso da FL foi identificado e em $10,5 \%$ a FL estava adjacente à localização sugerida pelo CTG , facilitando ainda, assim, determinação do local da fístula durante 0 ato cirúrgico. Os achados obtidos nesse trabalho são concordantes com os trabalhos de Mc Cormack ${ }^{21}$, Lant ${ }^{22}$ e Colquhoun. ${ }^{23}$

A detecção do contraste na imagem radiológica das cavidades nasais e paranasais é um dado extremamente específico para RLR. Schaefer et al..$^{24}$ sugeriram que se tenha uma TC nãocontrastada realizada imediatamente antes da realização da CTG, com a finalidade de se obter uma imagem de referência e evitar que algum artefato interfira no julgamento do exame.

Segundo Manefelde ${ }^{19}$, pode-se identificar o defeito ósseo na TC em $82 \%$ dos pacientes operados com FL; no entanto os números são discordantes em muitos outros trabalhos.

A glicose presente na RLR causa um edema da mucosa dos seios paranasais que pode ser documentado radiologicamente. ${ }^{5}$ No entanto esse dado carece de especificidade. Segundo Loyd et al. ${ }^{25}$, o achado do defeito ósseo e do aumento de partes moles ao seu redor é um dado altamente sensível para o diagnóstico de FL.

Em 1991, Wakhloo et al. ${ }^{12}$ sugeriram o uso da Ressonância Magnética associado à cisternotomografia; é necessário evidenciar que a RM permite a individualização do cérebro dos tecidos adjacentes, permitindo a demonstração de herniação do parênquima cerebral para o espaço extradural. ${ }^{9,10,26}$ Mais recentemente tem-se proposto o uso da RM cisternográfica, ponderada em T2, que dispensa o uso de contraste. Em nossa casuística usamos a RM apenas nos casos onde havia suspeita de meningoencefalocele ou de tumor intracraniano.

\section{CONCLUSÃO}

A cisternotomografia com meio de contraste nãoiônico hidrossolúvel Lopamidol ${ }^{\circledR}$ foi um método topodiagnóstico útil, com índices de detecção e localização similares aos descritos previamente na literatura para a CTG com metrizamida. A tomografia não-contrastada é útil como um método auxiliar da CTG, não devendo ser usada como método único no diagnóstico imaginológico da fístula liquórica.

\section{REFERÊNCIASBIBUOGRÁFICAS}

1. Willis T. Opera omnia: cerebri anatomia nervorumque descriptio et usus. Henri Westen, Amsterdam, 1782 apud Thompson St C. The cerebro-spinal fluid: its spontaneous escape from the nose. London: Cassell \& Co.; 1899. 140p.

2. Ommaya AK, Di Chiro G, Baldwin M, Pennybacker JB. Non traumatic cerebrospinal fluid rhinorrhea. J Neurol Neurosurg Psychiatry 1968; 31: 214-225.

3. Leech PJ, Paterson A. Conservative and operative management for cerebrospinal fluid leakage after closer head injury. Lancet 1973; 1(811):1013-6.

4. Lewin W. Cerebrospinal fluid rhinorrhea in closed head injuries. $\mathrm{Br}$ J Surg 1954; 42:1-18.

5. Guimarães RES. Localização de fístula liquórica da base anterior do crânio com o uso transoperatório de fluoresceína intratecal em solução hipodensa. Belo Horizonte, 2000, p. 16 (Tese de Doutorado - UFMG).
6. Gjuric M, Goede U, Keimer H, Wigand ME. Endonasal endoscopic closure of cerebrospinal fluid fistulas at the anterior cranial base. Ann Otol Rhinol Laryngol 1996; 105(8): 620-3.

7. Stammberger H, Greistofer K, Wolf G, Luxenberger W. Surgical occlusion of cerebrospinal fistulas of the anterior skull base using intrathecal sodium fluorescein. Laryngorhinootologie 1997; 76(10):595-607.

8. Chow JM, Goodman D, Mafee MF. Evaluation of CSF rhinorrhea by computerized tomography with metrizamide. Otolaryngol Head Neck Surg 1989; 100: 99-105.

9. Creamer MJ, Blendonohy P, Katz R, Russel E. Coronal computerized tomography and cerebrospinal fluid rhinorrhea. Arch Phys Med Rehabil 1992; 73(6): 599-602.

10. Jonhson DB, Brennan Toland J, O'dwyer AJ. Magnetic resonance imaging in the evaluation of cerebrospinal fluid fistulae. Clin Radiol 1996; 51(12) 837-41.

11. Toquet J, Bordure $P$, Herman P, Gayet-Delacroix $M$, Delaroche $O$, Legent $F$. Intérêt de la recherche de Beta2 transferrine et de la MR cisternographie pour le diagnostic des fistules de liquide céphalorachidien. Ann Otolaryngol Chir Cervicofac 1998; 115(5): 293-8.

12. Wakhloo AK, Van-Velthoven V, Schumacher M, Krauss JK Evaluation of MR imaging, digital subtraction cisternography, and CT cisternography in diagnosing CSF fistula. Acta Neurochir 1991; 111: 119-27.

13. Calcaterra TC. Extracranial surgical repair of cerebrospinal rhinorrhea. Ann Otol Rhinol Laryngol 1980; 89:108-16.

14. Hubbard JL, Mc Donald TJ, Pearson BW, Laws Jr ER. Spontaneous cerebrospinal fluid rhinorrhea: evolving concepts in diagnosis and surgical management based on the Mayo Clinic experience from 1970 through 1981. Neurosurgery 1985; 16(3): 314-21.

15. Meurman $\mathrm{OH}$, Irjala K, Suonpaa J, Laurent, B. A new method for the idenfication of cerebrospinal fluid leakage. Acta Otolaringol Stockh 1979; 87(3/4): 366-9.

16. Porter MJ, Brookes GB, Zeman AZ, Keir G. Use of protein electrophoresis in the diagnosis of cerebrospinal fluid rhinorrhea. J Laryngol Otol 1992; 106: 504-6.

17. Drayer BP, Wilkins RH, Boehnke M, Horton JA, Rosenbaum AE. Cerebrospinal fluid rhinorrhea demonstrated by metrizamide $\mathrm{CT}$ cisternography. AJR - Am J Roentgenol 1977; 129: 149-51.

18. Ghoshhaijra K. Metrizamide CT cisternography in the diagnosis and localization of cerebrospinal fluid rhinorrhea. J Comput Assist Tomogr 1980; 4: 306-10.

19. Manelfe C, Cellerier P, Sobel D, Prevost C, Bonafe A. Cerebrospinal fluid rhinorrhea:evolution with metrizamide cisternography. AJR - Am J Roentgenol 1982; 138: 471-6.

20. Naidich TP, Moran CJ. Precise anatomic localization of atraumatic sphenoethmoidal cerebrospinal fluid rhinorrhea by metrizamide CT cisternography. J Neurosurg 1980; 53: 222-8.

21. Mccormack B, Cooper PR, Persky M, Rothstein S. Extracranial repair of cerebrospinal fluid fistulas: technique and results in 37 patients. I Neurosurg 1990; 27: 412-7.

22. Lantz EJ, Forbes GS, Brown ML, Laws Jr ER. Radiology of cerebrospinal fluid rhinorrhea. AJR - Am J Roentgenol 1980; 135(5) 1023-30.

23. Colquhoun IR. CT cisternography in the investigation of cerebrospinal fluid rhinorrhea. Clin Radiol 1993; 47: 403-8.

24. Schaefer SD, Diehl J, Brigss WH. The diagnosis of CSF rhinorrhea by metrizamide CT scanning. Laryngoscope 1980; 90: 871-5.

25. Lloyd MNH, Kimber PM, Burrows EH. Post-Traumatic Cerebrospinal Fluid Rhinorrhea: Modern High-Definition Computed Tomography is All That is Required for the Effective Demonstration of the Site of Leakage. Clin Radiology 1994; 49:100-3.

26. Murata $Y$, Yamada I, Isotani E, Suzuki S. MRI in spontaneous cerebrospinal fluid rhinorrhea. Neuroradiology 1995; 37(6): 453-5.

27. Di Chiro G, Ommaya AK, Ashburm WL, Briner WH. Isotope cisternography in the diagnosis and follow-up of cerebrospinal fluid rhinorrhea. J Neurosurg 1968; 28(6): 522-9. 